\title{
OPERATIONS FOR THE RADICAL CURE OF INGUINAL HERNIA.
}

[Concluded from page 513, vol. 48.]

Practical plastic operations and the results of tenotomy, are suggestive as to the requirements for successful and permanent closure of apertures made within or upon the human body by accident or disease. The gap in a divided tendon is filled up, if reparatory action is excited, by a tissue identical or similar to the original growth-capable at least of performing the functions of the tissue to which the new growth is joined.

Sections of muscular fibres are repaired in like manner. The bond of union may differ in form and appearance; it may not possess the power of contractility, but is equal in strength, probably, to the muscle in the normal state, and the muscular function will afterwards be performed.

If action in a divided tissue by injury or disease is to be reparatory; something analogous to the tissue should be reproduced; and generally this is the fact, else upon what principle in fractured bones do surgeons rely upon bone deposit as a means of uniting firmly the separated parts. This union does not always occur; but the failure is the exception, and its cause not always understood.

Divided nerves may unite, and their functional properties return. This could not happen but in accordance with the law that each tissue reproduces its kind; and aponeuroses and tendons are not proved to be exempt from this general law, which complications and adverse influences may sometimes defeat.

When the "pillars of the ring" by pressure and hernial descent are dilated, and rendered flaccid, no spontaneous action restores them to a normal state, or forms any firm connection with the fasciæ, the muscular or other tissues which together constitute the natural barrier and boundaries of the inguinal canal.

A theory of cure, then, rests upon an hypothesis, which may be stated in a few words. If the pillars of the ring are wounded at particular points, a plastic, reparatory process will re-unite them to each other, to the external surfaces of the sac, or by new insertions to the pubic attachments; in either case lessening the dimensions of the tendinous bands, approximating their edges, and closing the abdominal ring.

There are other elements in the problem of cure besides incision or scarification of the tendinous structures; but upon this principle, successful operation is proposed, varying the manner in accordance with the characteristics of the case. The best means to accomplish the end may be considered when the operation is the subject of remark.

Radical cures of recent hernix caused by blows, have been noticed by surgeons, aided only by retentive means; it is reasonable to suppose the divided structures were united by the plastic process, and no departure from the usual mode of union is required to admit that in case of operation upon these structures there should be a similar result.

If in an operation for strangulation, the "pillars of the ring" had been purposely divided, and so placed as to reduce the ring to its normal size, and had become adherent, in such way that no hernia again appeared, would a theory of "radical cure" be considered extravagant or impossi- 
ble, which put forth this "innovation" in evidence of its support ? If radical cure followed such operation, would it be denied that this "new feature" had anything to do with this unusual result ?

Subcutaneous operation upon the "pillars of the ring" would facilitate plastic action, and be more reliable in cases where it is possible to effect a cure.

For reasons which need not be given, some herniæ do not admit of cure, even though the hypothesis was proved to be as sound as that one which proposed to restore vision by depressing an opaque lens, or that other which asserts its control over arterial bleeding by ligature properly applied to the divided vessel - whether caused by wounds or ruptured aneurismal sacs.

'The difference between the operations known, and that one suggested by the writer, will be seen if compared.

The usual mode is to excite inflammation in the sac, that adhesion may follow. In theory this operation should fail for the most part ; and it does so, according to the records of practice; for if the peritoneum in the normal state was incompetent to resist the protruding bowel with some support from fascix, tendons and natural adhesions, how should it, when the hernia is increased in bulk, and the pressure is greater, accomplish retention when attenuated firmer structures, and dilated pillars of the ring, fail to give the support supplied in their normal condition and in natural association?

By a new method it is assumed that plastic action may be induced by proper means in the tendinous structures, without "inflammation ;" and in cases where the stricture is not at the external ring in strangulation, if the "pillars of the ring" are incised and approximated by suture if necessary, after the usual operation for relief of strangulation, a radical cure will be a probable result.

Here the risk attendant upon inflammation is increased, which might embarrass curative action. But inasmuch as it is proved that plastic action, on the modelling process, proceeds with more certainty under cover, the "pillars of the ring," or other tissues acted upon from a distance by subcutaneous manipulation, would be a preferable mode, Dr. Heaton says he "has discovered a safe and simple way," for the approximation of the pillars and closure of the abdomianl ring. Will his practice verify or disprove the advanced hypothesis? As to the safety of the operation, little need be said. Its successful issue would much depend upon the precision and skill of the operator.

It was not my design to compose an essay on the operations for the cure of hernia, ancient or modern, and I may have occupied more space than I intended in this imperfect outline of a subject, interesting to myself if not to the majority of medical readers. I do not know that $I$ have recorded an original idea, and it may be proven to me that it is a correct deduction " to cure hernia by producing adhesive inflammation of the walls of the sac." The proposed hypothesis of tendinous union may have been tried or attempted by many operators, and found impracticable either in the operation or in its results. My limited acquaintance with medical literature does not, however, furnish me with any evidence 
upon this point. I incline to the opinion that the "pillars of the ring" have been wounded in operations, or irritated by pressure, producing beneficial results and cures, without the knowledge of the operator, and the action thus induced has had the principal agency in the cures.

I coincide with those physiologists and pathologists who believe that inflammation is not normal action, nor necessary in any reparatory process of the animal organs, or tissues, and that according to the time and degree of its obtrusion in cases of injury or disease, it retards the cure, sometimes destroying the vitality of a part or organ, and as a consequence suspension of all the functions necessary to life.

Is it sound doctrine to rely for cure upon the production of an action no more required to form one cell, which is to become a part of a living normal tissue, then it is required in the act of generation, to vitalize and set in motion a germ which is destined, according to natural laws, to become the representative of the highest order of the created things of the earth.

No. 1 Bowdoin st., Baston, July, 1853.

\section{AN ADDITIONAL MUSClE OF THE EYE.}

BY N. R. MOSELEY, M.D., PHILADELPHIA.

[Communicated for the Boston Medical and Surgical Journal.]

By dissections made recently by myself of the tissues within the orbits of the human eye, I have found a muscle that I never met with before, and a description of which I have not seen in the books. The muscle referred to is a small mass placed upon the outer side of the globe, running parallel for a short distance with the m. abducens. The fibres take origin from the orbital surface of the malar bone anterior to the point of union of this bone with the orbital surface of the sphenoid bone; from thence running forward, its tendon blends with the fibrous structure of the outer angle of the eyelid. By making the muscle tense, the outer canthus is drawn outward and backward. 'Thus it is an antagonist of the Tensor Tarsi of Horner. Now whether this muscle is always present in the human subject or not, I am unable to say; but by several wet specimens in my possession I can satisfactorily demonstrate it, be it anomalous or otherwise.

July 17, 1853.

\section{MECHANICAL CURE OF SPERMATORRHCEA.}

To the Editor of the Boston Medical and Surgical Journal.

THE advantages of reading medical journals are great. Yours has been received with great regularity for five years, and each number thoroughly read. It contains many valuable and practical suggestions. For example, an article on spermatorrhœa, June 29th, was read with interest. The next day a patient with that difficulty applied for relief. It was decided to pursue the plan described in the article alluded to. A piece 